\title{
Bacterial communities associated with cell phones and shoes
}

\author{
David A Coil ${ }^{\text {Corresp., } 1}$, Russell Y Neches ${ }^{1}$, Jenna M Lang ${ }^{1}$, Guillaume Jospin ${ }^{1}$, Wendy E Brown ${ }^{2,3}$, Darlene Cavalier ${ }^{3,4}$, \\ Jarrad Hampton-Marcell ${ }^{5}$, Jack A Gilbert ${ }^{6}$, Jonathan A Eisen ${ }^{\text {Corresp. } 7}$ \\ ${ }^{1}$ Genome Center, University of California, Davis, Davis, United States \\ 2 Department of Biomedical Engineering, University of California, Irvine, Irvine, California, United States \\ 3 Science Cheerleaders, Inc, Irvine, United States \\ 4 SciStarter.org, Irvine, United States \\ 5 Argonne National Laboratory, University of Chicago, Lemont, Illinois, United States \\ 6 Department of Pediatrics and Scripps Institution of Oceanography, UC San Diego School of Medicine, San Diego, California, United States \\ 7 Genome Center, Department of Evolution and Ecology, Department of Medical Microbiology and Immunology, University of California, Davis, Davis, \\ California, United States \\ Corresponding Authors: David A Coil, Jonathan A Eisen \\ Email address: dcoil@ucdavis.edu, jaeisen@ucdavis.edu
}

Background: Every human being carries with them a collection of microbes, a collection that is likely both unique to that person, but also dynamic as a result of significant flux with the surrounding environment. The interaction of the human microbiome (i.e., the microbes that are found directly in contact with a person in places such as the gut, mouth, and skin) and the microbiome of accessory objects (e.g., shoes, clothing, phones, jewelry) is of potential interest to both epidemiology and the developing field of microbial forensics. Therefore, the microbiome of personal accessories are of interest because they serve as both a microbial source and sink for an individual, they may provide information about the microbial exposure experienced by an individual, and they can be sampled non-invasively.

Findings: We report here a large-scale study of the microbiota found on cell phones and shoes. Cell phones serve as a potential source and sink for skin and oral microbiota, while shoes can act as sampling devices for microbial environmental experience. Using 16S rRNA gene sequencing, we characterized the microbiota of thousands of paired sets of cell phones and shoes from individuals at sporting events, museums, and other venues around the United States.

Conclusions: We place this data in the context of previous studies and demonstrate that the microbiota of phones and shoes are different. This difference is driven largely by the presence of "environmental" taxa (taxa from groups that tend to be found in places like soil) on shoes and human-associated taxa (taxa from groups that are abundant in the human microbiome) on phones. This large dataset also contains many novel taxa, highlighting the fact that much of microbial diversity remains uncharacterized, even on commonplace objects. 
1

2

3

4

5

6

7

8

9 10

11

12

13

14

15

26

27

28

29

30

31

32

33

34

35

36

\section{Bacterial communities associated with cell phones and shoes}

David A. Coil, Russell Y. Neches, Jenna M. Lang, Guillaume Jospin, Wendy E. Brown, Darlene Cavalier, Jarrad Hampton-Marcell, Jack A. Gilbert, Jonathan A. Eisen

David A. Coil (coil.david@gmail.com). Genome Center, University of California, Davis, CA, USA

Russell Y. Neches (russell@vort.org). Genome Center, University of California, Davis, CA, USA

Jenna M. Lang (jennomics@gmail.com) Genome Center, University of California, Davis, CA, USA

Guillaume Jospin (guillaume.jospin@gmail.com). Genome Center, University of California, Davis, CA, USA

Wendy E. Brown (wendy.brown@uci.edu). Science Cheerleaders, Inc., Department of Biomedical Engineering, University of California Irvine, Irvine, CA, USA

Darlene Cavalier (darcav1@gmail.com). Science Cheerleaders, Inc., Irvine, SciStarter.org, United States

Jarrad Marcell (jhampton-marcell@anl.gov). Argonne National Laboratory, University of Chicago, Lemont, IL, USA

Jack A. Gilbert (gilbert.jack@gmail.com). Department of Pediatrics and Scripps Institution of Oceanography, UC San Diego School of Medicine, San Diego, CA, USA

Jonathan A. Eisen (jaeisen@ucdavis.edu). Genome Center, Department of Evolution and Ecology, Department of Medical Microbiology and Immunology, University of California, Davis, Davis, CA, USA

Corresponding Authors:

David A. Coil

451 Health Sciences Drive, Davis, CA, 95618, USA

Email address: coil.david@gmail.com

Jonathan A. Eisen

451 Health Sciences Drive, Davis, CA, 95618, USA

Email address: jaeisen@ucdavis.edu 
37

38

39

40

41

42

43

44

45

46

47

48

49

50

51

52

53

54

55

56

57

58

59

60

61

62

63

64

65

66

67

68

69

70

71

72

73

74

75

76
Running title: Cell phone and shoe bacteria

\begin{abstract}
Background: Every human being carries with them a collection of microbes, a collection that is likely both unique to that person, but also dynamic as a result of significant flux with the surrounding environment. The interaction of the human microbiome (i.e., the microbes that are found directly in contact with a person in places such as the gut, mouth, and skin) and the microbiome of accessory objects (e.g., shoes, clothing, phones, jewelry) is of potential interest to both epidemiology and the developing field of microbial forensics. Therefore, the microbiome of personal accessories are of interest because they serve as both a microbial source and sink for an individual, they may provide information about the microbial exposure experienced by an individual, and they can be sampled non-invasively.
\end{abstract}

Findings: We report here a large-scale study of the microbiota found on cell phones and shoes. Cell phones serve as a potential source and sink for skin and oral microbiota, while shoes can act as sampling devices for microbial environmental experience. Using 16S rRNA gene sequencing, we characterized the microbiota of thousands of paired sets of cell phones and shoes from individuals at sporting events, museums, and other venues around the United States.

Conclusions: We place this data in the context of previous studies and demonstrate that the microbiota of phones and shoes are different. This difference is driven largely by the presence of "environmental" taxa (taxa from groups that tend to be found in places like soil) on shoes and human-associated taxa (taxa from groups that are abundant in the human microbiome) on phones. This large dataset also contains many novel taxa, highlighting the fact that much of microbial diversity remains uncharacterized, even on commonplace objects.

\title{
Keywords
}

16S rRNA gene, cell phones, shoes, citizen science, biogeography, human microbiome, Illumina, taxonomy, microbial dark matter, ASV

\section{Introduction}

Our understanding of the human microbiome (e.g. (McDonald et al., 2018)), the microbiome of the built environment around us (e.g. (National Academies of Sciences, Engineering, and Medicine et al., 2017)), and the interactions between the two (e.g. (Leung \& Lee, 2016)) have dramatically expanded in recent years. This understanding has implications for fields ranging 
77 from medicine to forensics to architecture. In addition to the millions of microbes that we carry

78

79

80

81

82

83

84

85

86

87

88

89

90

91

92

93

94

95

96

97

98

99

100

101

102

103

104

105

106

107

108

109

110

111

112

113

114

115

116

around each day, the majority of people on the planet are thought to now possess a cell phone.

Previous work on the microbiome associated with phones has shown that people share a much greater percentage of their microbes with their own phone than with the phones of others (Meadow, Altrichter \& Green, 2014). Additionally, these authors showed a high correlation between the index finger specifically, and the surface of the owner's phone. As for the environment around us, shoes (or other foot coverings) can act as microbial sampling devices. We have previously described data suggesting this to be the case, as well as demonstrated that the microbiome of cell phones and shoes from the same person are quite distinct (Lax et al., 2015).

Though the existence of microbes has been known for a few hundred years, only in recent decades have we come to learn of the existence of the many microorganisms on the planet that have not yet been cultivated in a lab. This so-called "microbial dark matter" (MDM) is understudied and probably makes up a majority of microbial life on the planet (Solden, Lloyd \& Wrighton, 2016; Bernard et al., 2018; Lloyd et al., 2018). Sometimes the term refers to any uncultured taxa, while others use it to refer to major evolutionary lineages for which few or no representatives have ever been grown in the lab or studied in detail (Rinke et al., 2013). Here we use "MDM" in the latter, more general sense. While MDM taxa are probably best known from extreme environments like acid mine drainage and the deep sea, there are presumably also many relatively unknown taxa on items as commonplace as phones and shoes.

Throughout 2013-2014, we organized public events around the United States for the purpose of swabbing surfaces of the built environment and collecting bacteria for isolation via culturing. Cultured isolates from these samples were screened and a subset of them were sent to the International Space Station (ISS) for growth in microgravity (Coil et al., 2016). As part of the public outreach component of this project, we engaged the public in helping collect these swabs, as well as in swabbing their cell phones and shoes for a nationwide microbial biogeography study. Thousands of people participated in this project, and we initially collected $\sim 3,500$ paired cell phone/shoe samples, of which we sequenced $\sim 2,500$ samples. The intent of examining bacteria on cell phones and shoes was threefold; firstly to scale up the results of previous studies on shoes and phones and to look for patterns in the biogeography at a national scale. The second was to engage people in thinking about cell phones as being a putative proxy for sampling the microbes found on a person and their shoes as being a putative proxy for sampling the microbes found in a person's environment. Lastly, we wanted to search for MDM taxa on common, human-associated objects. To our knowledge, this represents the largest collection of bacterial community sequencing data associated with cell phones or shoes.

\section{Materials and Methods}

PeerJ reviewing PDF | (2019:01:34673:2:0:NEW 29 Apr 2020) 


\section{Sample collection}

118

119

120

121

122

123

124

125

126

127

128

129

130

131

132

133

134

135

136

137

138

139

140

141

142

143

144

145

146

147

148

149

150

151

152

153

154

155

156

Cell phone and shoe samples were collected on sterile cotton swabs (Puritan cotton tipped \#25806) and participants were instructed to "swab for about 15 seconds as if trying to clean the object". Swabs were kept at room temperature by necessity and then sent overnight to the University of Chicago, where they were kept at $-80^{\circ} \mathrm{C}$ until processing. Metadata for the samples included the physical location (GPS coordinates), date of sampling, rough age of participants, sample object type (cell phone or shoe), and event (basketball game, museum visit, etc.). Participant age was estimated by the event organizers as primarily children (e.g. an elementary school), primarily adults (e.g. a conference), or a mix (e.g. a baseball game). This study was performed under an expedited review and waiver through the University of Chicago IRB under protocol 'Phones and Shoes Study' IRB13-1091 awarded to Jack Gilbert.

\section{DNA extraction and Sequencing}

DNA extractions, library preparation, and Illumina sequencing (paired-end $150 \mathrm{bp}$ ) were performed exactly as described in our previous work using swabs from the ISS (Lang et al., 2017). In brief: samples were prepared using Mo BIO UltraClean kits, DNA extracted using Zymo ZR-96 kits, DNA amplified using EMP barcoded primer sets targeting the V4 region of the 16S rRNA gene, amplicons were cleaned and pooled and sequenced on an Illumina MiSeq platform.

\section{Data processing, validation and generation of ASV tables}

The dataset (2,486 sequenced samples) was prepared by following the DADA2 protocol ("big data") (Callahan et al., 2016a) to generate amplicon sequence variants (ASVs). Each sequencing lane was also pre-processed individually to account for error patterns from different runs or machines. Reads longer than 150 base pairs (bp) were trimmed down to $150 \mathrm{bp}$ before processing with DADA2. Low quality regions of reads were removed by trimming bases that did not satisfy a Q2 quality score. The reads were also trimmed down to a length of $145 \mathrm{bp}$. Reads containing Ns were discarded and we used two expected errors to filter the overall quality of the read (rather than averaging quality scores) (Edgar \& Flyvbjerg, 2015). Only forward reads were considered for this study, in order to be consistent with previous work. Quality filtering resulted in 2,230 samples being analyzed. 186,334 unique ASVs were identified and taxonomic assignments were made for these ASVs using the Silva NR v132 database. Samples without complete metadata were excluded. Using Phyloseq, the non-bacterial ASVs that were assigned to mitochondria or chloroplasts (in total 63,838 or $34 \%$ of the ASVs) were excluded from further analysis, resulting in 148,535 remaining ASVs. The ASV based filtration reduced the total number of samples to 2,230 (since some samples did not contain any of these final ASVs). After rarefying to $10 \mathrm{k}$ reads per sample, 44,897 ASVs were no longer represented in the data set and 
157348 samples were removed due to insufficient ASVs. In total, 17,550,000 of the initial reads

158

159

160

161

162

163

164

165

166

167

168

169

170

171

172

173

174

175

176

177

178

179

180

181

182

183

184

185

186

187

188

189

190

191

192

193

194

195

were used for the Alpha diversity analyses. The data was additionally filtered to only include ASVs present in $>5 \%$ of the samples and rarefied again to $10 \mathrm{k}$ reads per samples which resulted in 2,253 ASVs for 1,672 samples. This version was used in the beta diversity analyses. For Alpha diversity, additional filtering was required, pairing 637 phone samples to shoe samples (totalling 1,274). For the biogeography analysis, samples were summed by Event which resulted in the exclusion of five events, resulting in 34 Event locations being evaluated after the previous pairing step. A table of all filtering/processing steps can be found as Supplemental Table 1.

\section{Diversity analyses (alpha, beta, taxonomic, phylogenetic)}

Alignment of the observed sequences was performed using Clustal Omega (Goujon et al., 2010; Sievers et al., 2011), and an approximate maximum likelihood phylogeny was constructed using FastTree2 (Price, Dehal \& Arkin, 2009, 2010). Metadata was loaded from the mapping files and relevant columns were extracted using Pandas (McKinney \& Others, 2010) (retained values were: Age, City, Date, Event, Run, Sample, Sport, State, Type). ASV filtering, taxonomic agglomeration, and ordination was performed using phyloseq (McMurdie \& Holmes, 2013) using Callahan et al. as a guide (Callahan et al., 2016b).

The alpha diversity metrics were calculated using phyloseq and ggplot $\mathrm{R}$ packages as well as a reduced dataset in which we removed all "Sample: Unknown" samples. We then rarified the samples to 10,000 reads. Only the samples which had corresponding phone and shoe pairs were considered for plotting the Shannon and Observed diversity metrics. We chose 10,000 reads for a rarefaction cut off by plotting all sample's rarefaction curves and picked a cutoff that would balance sample inclusion and enough ASVs. The PCoA ordination of the Bray-Curtis distances of the ASV data was generated using the ordinate and plot_ordination functions from Phyloseq. As input to the ordination function, we further filtered the ASVs to those represented in at least $5 \%$ of the samples then rarefied to $10 \mathrm{k}$ reads per samples. We exported the ordination coordinates and averaged values for cell phones and shoes separately to find the centroid of the two data spreads. We plotted a line bisecting perpendicularly the segment between the two centroids to highlight the separation between the two groups. We used ggplot2 to overlay this line on the sample and taxa (at the phylum level) versions of the PCoA (Wickham, 2010). We ran an ANalysis Of SIMilarity (ANOSIM) test available through the vegan R package to assess the similarities between the phone and shoe samples using Bray-Curtis distances and 999 permutations (Oksanen et al., 2011).

We plotted (ggplot2) the Bray-Curtis distances (vegan) on the ASV counts of the samples summed by sampling sites (phyloseq) against the physical distances between the sites. We used a custom perl script to calculate the geographical distances using GPS coordinates and making the 
196

197

198

199

200

201

202

203

204

205

206

207

208

209

210

211

212

213

214

215

216

217

218

219

220

221

222

223

224

225

226

227

228

229

230

231

232

233

234

235

assumption that the Earth is a sphere. Mantel tests were done using the ade4 (Dray \& Dufour, 2007) R package.

\section{Attribute Importance Analysis}

Random forest and related analyses were done using the scikit-learn v0.21.2 Python package (Pedregosa et al., 2011). Variable importance measures were estimated by first training the random forest classifier (Breiman, 2001; Geurts, Ernst \& Wehenkel, 2006; Pedregosa et al., 2011) on the final ASV counts and then extracting the attribute importance values, also called the gini importances or mean decrease impurity (Breiman, Friedman, Olshen \& Stone, 1984) from the trained classifiers (Janitza, Strobl \& Boulesteix, 2013). Other than specifying 50 estimators, the default parameters were used. The figure was generated using the matplotlib Python package (Hunter, 2007).

\section{Results/Discussion}

\section{Alpha Diversity}

In total, 3,500 swabs were collected for this study at 38 events (see Supplemental Table 2 for details on events). Of these, some samples were lost in transit and a further 864 samples were excluded from sequencing due to an irretrievable loss of the sample ID data (computer failure). The exact number of actual swabs originally collected/lost is unknown, due to the distributed nature of the collection as part of a citizen science project. Sequencing was performed on 2,486 samples with 599,386,254 paired end reads generated across four lanes of Illumina HiSeq PE150.

To examine the alpha diversity of these samples, we examined all pairs of samples where both the cell phone and the shoe had at least 10,000 reads. The plot of both observed counts and the Shannon diversity index $(\mathrm{H})$ can be seen in Figure 1. By either measure, shoes have a significantly higher alpha diversity than phones. This is concordant with previous results and presumably results from the greater variety of environmental taxa that shoes might encounter over time.

\section{Attribute Importance}

As a method for examining the potential importance of the metadata variables (sample type, sport, location, and sequencing run), we utilized variable importance measures (VIMs). These 
236 VIMs were estimated by training a random forest classifier (Breiman, 2001; Geurts, Ernst \& 237 Wehenkel, 2006; Pedregosa et al., 2011) to assign samples to their metadata categories (sample 238 type, city, state, sequencing run, and sport) based on their ASV counts, and extracting the 239 variable importance values (Breiman, 2017) from the trained classifiers (Janitza, Strobl \& 240 Boulesteix, 2013). VIMs are implemented as the total decrease in node impurity, weighted by the 241 probability of reaching that node as approximated by the proportion of samples reaching that 242 node, averaged over all trees in the ensemble

243 (https://stackoverflow.com/questions/15810339/how-are-feature-importances-inrandomforestclassifier-determined). Note that variable importance analysis is a distinct application of random forests from the more widely-used classification application. Extracting VIMs does not include the optimization and benchmarking steps required to use random forests in their predictive capacity. Sample feature importances indicate that the sample type (shoe or phone) was the most predictive of the observed community structure, followed by the geographic location of the sample (Supplemental Figure 1). The sport played at the venue where the sample was collected is less predictive of the community structure than the sequencing run. Overall, these results support and extend our previous findings that the microbiomes of shoes and phones are distinct. Interestingly, the city where an event took place was more predictive of community

253

254

255

256

257

258

259

260

261

262

263

264

265

266

267

268

269

270

271

272

273

274

275 structure than state, suggesting the possibility that there are local biogeography effects in patterning the microbial community.

\section{Beta Diversity}

In order to examine and visualize differences between samples, we plotted a PCoA ordination of samples based on sample to sample Bray-Curtis distances of the rarefied microbial communities that appear in more than 5\% of the samples (Figure 2). A quick examination of the plot revealed that cell phones (green) and shoes (black) appear to group separately (something seen in prior studies); this is supported by statistical analysis (ANOSIM $\mathrm{R}=0.5736, \mathrm{p}=.001$ ).

To further examine the differences between cell phones and shoes, we identified the centroids of the two data spreads (Figure 2). The line in this figure represents the bisection of these two centroids, to highlight their separation. We then used this bisection line to examine in more detail the taxa that contribute to the separation of shoe and phone samples. We did this by generating a series of plots showing only the ASVs belonging to each phylum separately (Figure 3), showing only those that were significant in our ANCOM analysis. The line in each plot is the same as in the sample plot in Figure 2 and those ASVs to the top/left can be considered to be driving the "phone" portion of the PCoA and the ASVs to the bottom/right can be considered to drive the "shoe" portion of the PCoA. These plots (and the underlying data) show some interesting phylaspecific patterns. Some phyla (e.g., Bacteroides and Firmicutes) have many ASVs on both sides of the line, indicating that there are ASVs from these phyla that are significantly biased towards shoes and others that are significantly biased towards phones. 
277 One phylum (Fusobacteria) contains only ASVs that are skewed towards phones. We believe this

278

279

280

281

282

283

284

285

286

287

288

289

290

291

292

293

294

295

296

297

298

299

300

301

302

303

304

305

306

307

308

309

310

311

312

313

314

315

is likely due to these ASVs being human associated taxa. For example, the taxonomic assignments of the Fusobacteria ASVs were Leptotrichia $(\mathrm{n}=2)$ and Fusobacterium $(\mathrm{n}=1)$; these two genera are generally found in animal microbiomes including the oral microbiome of humans and other mammals (Eribe \& Olsen, 2008; Whitman et al., 2015a,b). On the other hand, there are two phyla (Deinococcus-Thermus, Cyanobacteria) which include only ASVs that are skewed towards shoes. We presume that these ASVs from these phyla represent taxa from the broader environment (e.g., soil) that would be picked up by shoes. Examination of the taxonomic assignments for these ASVs supports this possibility, with genera assignments including taxa commonly found in water or soil such as Chroococcidiopsis e.g. (Billi et al., 2000), Oscillatoria e.g. (Carpenter \& Price, 1976), Truepera e.g. (Albuquerque et al., 2005), and Deinococcus e.g. (Battista, Earl \& Park, 1999).

Biogeography

This study included sampling sites as close together as within the same city (e.g. multiple events in Philadelphia, PA) as well as sites spread out across the United States. Previous biogeography work on a continental scale (China) showed that environmental bacteria had a strong relationship between community similarity and geographic distance, while Archaea showed no such pattern (Ma et al., 2017). We conducted a similar analysis, treating both cell phone and phone samples separately (Figure 4). Both cell phones and shoes are very "noisy" in this analysis, some samples that are within the same city have radically different communities and some samples thousands of miles apart have very similar bacterial communities. Therefore, we do not observe a significant correlation between community similarity and geographic distance, in either cell phones or shoes.

Novel evolutionary lineages

Additionally, we examined how many (if any) of these microbes present on cell phones and shoes were from any of the so-called "microbial dark matter" branches in the tree of life. The term "microbial dark matter" or MDM for short is used in this context to refer to major evolutionary lineages for which few or no representatives have ever been grown in the lab or studied in detail (Rinke et al., 2013).

To identify MDM in our data, we searched through the taxonomic annotation of ASVs for those assigned to phyla or candidate phyla which are generally viewed as MDM lineages. Specifically, we considered ASVs assigned to the following groups as being MDM: Aegiribacteria, AncK6, Armatimonadetes, Atribacteria, BRC1, Caldiserica, Calditrichaeota, Chrysiogenetes, Cloacimonetes, Coprothermobacteraeota, Dadabacteria, Dependentiae, Diapherotrites, 
316 Edwardsbacteria, Elusimicrobia, Entotheonellaeota, Fervidibacteria, FCPU426, GAL15,

317 Hydrogenedentes, Latescibacteria, Margulisbacteria, Nanoarchaeaeota, Nitrospinae,

318 Omnitrophicaeota, Patescibacteria, PAUC34f, Rokubacteria, RsaHf231, WOR-1, WPS-2, WS1,

319 WS2, WS4, and Zixibacteria. We chose these groups because of all the phyla to which our ASVs

320 were assigned, these are the groups that either contain no cultured representatives or for which

321 most of the phylogenetic diversity within the group is only represented by uncultured taxa. We

322 also then examined the distribution patterns of these ASVs across samples and whether they

323 showed any skew between phones and shoes (Supplemental Table 3).

324

325

This analysis of ASVs assigned to MDM lineages revealed that, in fact, quite a large number of

326

327

328

329

330

331

332

333

334

335

336

337

338

339

340

341

342

343

344

345

346

347

348

349

350

351

352

353

354

355 ASVs found in our study were from such MDM groups. In some cases, these ASVs assigned to these groups are quite rare - for example, ASVs from WOR-1, Edwardsbacteria, and Diapherotrites were found to be present in one sample each. However, some were present in a much wider range of samples, and we focused most of our attention on those (Supplemental Table 3). Of the nine MDM phyla for which ASVs were found to be present in at least $10 \%$ of samples (Armatimonadetes, Patescibacteriam, WPS-2, Entotheonellaeota, Dependentiae, BRC1, Rokubacteria, Latescibacteria, Elusimicrobia), all were found more often in shoe samples than phone samples. This is not surprising given that (1) phone samples tend to be enriched for human associated microbes, only a few of which are in current MDM groups and (2) many MDM lineages are known to be found in soil, which is presumably abundant on shoes. Two of these widespread MDM groups (Armatimonadetes, Patescibacteria) were found to have ASVs present in almost $50 \%$ of samples. The Armatimonadetes phyla is known to be both diverse and widespread, with soil contributing the most members of this group of any single environment (Lee, Dunfield \& Stott, 2014). The proposed Patescibacteria superphylum also contains a wide variety of diverse taxa, but the majority are associated with aquatic or semi-aquatic environments (Sánchez-Osuna, Barbé \& Erill, 2017). Twelve classes and thirteen orders were found to be present in more than $10 \%$ of samples. Of these, all were skewed towards shoe samples, except two taxa (Gracilibacteria within Patescibacteria and Absconditabacteriales within Gracilibacteria).

Overall, these results show that, while MDM might be thought of as coming from remote, isolated, or extreme environments, a remarkable fraction of people are traveling around with representatives from these uncultured groups on commonplace objects. This highlights how much we still have to learn about the microbial world around us.

\section{Summary}

These data support previous work by ourselves and others demonstrating that the microbiome of cell phones and shoes are distinct, even when belonging to the same person. The taxonomic diversity of shoes appears to be much higher than that of phones. In this analysis, we also 
356 highlight which phyla are most responsible for the observed differences in microbial 357 communities between phones and shoes. This difference is driven largely by the presence of 358 "environmental" taxa (taxa from groups that tend to be found in places like soil) on shoes and 359 human-associated taxa (taxa from groups that are abundant in the human microbiome) on 360 phones. We did not observe a correlation between geographic distance and community 361 similarity. Lastly, we show that a number of "microbial dark matter" taxa are present, even 362 abundant, on these commonplace objects.

363

364

365

366

367

368

369

370

371

372

373

374

375

376

377

378

379

380

381

382

383

384

385

386

387

388

389

390

\section{Availability of Supporting Data}

All raw sequencing data has been deposited at NCBI under BioProject PRJNA470730

(https://www.ncbi.nlm.nih.gov/sra/SRP145522). All data analysis, metadata, supporting files and intermediate analysis files are available at Zenodo: (https://zenodo.org/record/3665855). An interactive visualization of this data is available at www.phinch.org. A website describing this project and related ones can be found at www.spacemicrobes.org.

\section{Acknowledgements}

The authors would like to thank the many Science Cheerleaders who ran the sampling events. In particular, we'd like to thank Bart Leahy who coordinated the coordinators. We would also like to thank SciStarter and Science Cheerleaders, Inc. for being partners in this work. Thanks to Bretwood Higman for the script used to determine geographic distances. And finally, we would like to thank the thousands of people who gave willingly of their time and their samples as part of participating in this study.

\section{References}

Albuquerque L, Simões C, Nobre MF, Pino NM, Battista JR, Silva MT, Rainey FA, da Costa MS. 2005. Truepera radiovictrix gen. nov., sp. nov., a new radiation resistant species and the proposal of Trueperaceae fam. nov. FEMS microbiology letters 247:161-169.

Battista JR, Earl AM, Park MJ. 1999. Why is Deinococcus radiodurans so resistant to ionizing radiation? Trends in microbiology 7:362-365.

Bernard G, Pathmanathan JS, Lannes R, Lopez P, Bapteste E. 2018. Microbial Dark Matter 
391

392

393

394

395

396

397

398

399

400

401

402

403

404

405

406

407

408

409

410

411

412

413

Investigations: How Microbial Studies Transform Biological Knowledge and Empirically

Sketch a Logic of Scientific Discovery. Genome biology and evolution 10:707-715.

Billi D, Friedmann EI, Hofer KG, Caiola MG, Ocampo-Friedmann R. 2000. Ionizing-radiation resistance in the desiccation-tolerant cyanobacterium Chroococcidiopsis. Applied and environmental microbiology 66:1489-1492.

Breiman L. 2001. Random Forests. Machine learning 45:5-32.

Breiman L. 2017. Classification and regression trees. Routledge.

Callahan BJ, McMurdie PJ, Rosen MJ, Han AW, Johnson AJA, Holmes SP. 2016a. DADA2: High-resolution sample inference from Illumina amplicon data. Nature methods 13:581583.

Callahan BJ, Sankaran K, Fukuyama JA, McMurdie PJ, Holmes SP. 2016b. Bioconductor Workflow for Microbiome Data Analysis: from raw reads to community analyses. F1000Research 5:1492.

Carpenter EJ, Price CC. 1976. Marine oscillatoria (Trichodesmium): explanation for aerobic nitrogen fixation without heterocysts. Science 191:1278-1280.

Coil DA, Neches RY, Lang JM, Brown WE, Severance M, Cavalier D, Eisen JA. 2016. Growth of 48 built environment bacterial isolates on board the International Space Station (ISS). PeerJ 4:e1842.

Dray S, Dufour A-B. 2007. The ade4 Package: Implementing the Duality Diagram for Ecologists. Journal of Statistical Software, Articles 22:1-20.

Edgar RC, Flyvbjerg H. 2015. Error filtering, pair assembly and error correction for nextgeneration sequencing reads. Bioinformatics 31:3476-3482.

Eribe ERK, Olsen I. 2008. Leptotrichia species in human infections. Anaerobe 14:131-137. 
414 Geurts P, Ernst D, Wehenkel L. 2006. Extremely randomized trees. Machine learning 63:3-42.

415 Goujon M, McWilliam H, Li W, Valentin F, Squizzato S, Paern J, Lopez R. 2010. A new

416 bioinformatics analysis tools framework at EMBL-EBI. Nucleic acids research 38:W695-

$417 \quad$ W699.

418 Hunter JD. 2007. Matplotlib: A 2D Graphics Environment. Computing in Science Engineering

419 9:90-95.

420 Janitza S, Strobl C, Boulesteix A-L. 2013. An AUC-based permutation variable importance

421 measure for random forests. BMC bioinformatics 14:119.

422 Lang JM, Coil DA, Neches RY, Brown WE, Cavalier D, Severance M, Hampton-Marcell JT,

423 Gilbert JA, Eisen JA. 2017. A microbial survey of the International Space Station (ISS).

$424 \quad$ PeerJ 5:e4029.

425 Lax S, Hampton-Marcell JT, Gibbons SM, Colares GB, Smith D, Eisen JA, Gilbert JA. 2015.

426 Forensic analysis of the microbiome of phones and shoes. Microbiome 3:21.

427 Lee KCY, Dunfield PF, Stott MB. 2014. The Phylum Armatimonadetes. In: Rosenberg E,

428 DeLong EF, Lory S, Stackebrandt E, Thompson F eds. The Prokaryotes: Other Major

429 Lineages of Bacteria and The Archaea. Berlin, Heidelberg: Springer Berlin Heidelberg,

$430 \quad 447-458$.

431 Leung MHY, Lee PKH. 2016. The roles of the outdoors and occupants in contributing to a

432 potential pan-microbiome of the built environment: a review. Microbiome 4:21.

433 Lloyd KG, Steen AD, Ladau J, Yin J, Crosby L. 2018. Phylogenetically Novel Uncultured

434 Microbial Cells Dominate Earth Microbiomes. mSystems 3. DOI:

$435 \quad 10.1128 / \mathrm{mSystems.00055-18.}$

436 Ma B, Dai Z, Wang H, Dsouza M, Liu X, He Y, Wu J, Rodrigues JLM, Gilbert JA, Brookes PC, 
437

438

439

440

441

442

443

444

445

446

447

448

449

450

451

452

453

454

455

456

457

458

459

Xu J. 2017. Distinct Biogeographic Patterns for Archaea, Bacteria, and Fungi along the Vegetation Gradient at the Continental Scale in Eastern China. mSystems 2. DOI: 10.1128/mSystems.00174-16.

McDonald D, Hyde E, Debelius JW, Morton JT, Gonzalez A, Ackermann G, Aksenov AA, Behsaz B, Brennan C, Chen Y, DeRight Goldasich L, Dorrestein PC, Dunn RR, Fahimipour AK, Gaffney J, Gilbert JA, Gogul G, Green JL, Hugenholtz P, Humphrey G, Huttenhower

C, Jackson MA, Janssen S, Jeste DV, Jiang L, Kelley ST, Knights D, Kosciolek T, Ladau J, Leach J, Marotz C, Meleshko D, Melnik AV, Metcalf JL, Mohimani H, Montassier E, Navas-Molina J, Nguyen TT, Peddada S, Pevzner P, Pollard KS, Rahnavard G, RobbinsPianka A, Sangwan N, Shorenstein J, Smarr L, Song SJ, Spector T, Swafford AD, Thackray VG, Thompson LR, Tripathi A, Vázquez-Baeza Y, Vrbanac A, Wischmeyer P, Wolfe E, Zhu Q, American Gut Consortium, Knight R. 2018. American Gut: an Open Platform for Citizen Science Microbiome Research. mSystems 3. DOI: 10.1128/mSystems.00031-18. McKinney W, Others. 2010. Data structures for statistical computing in python. In: Proceedings of the 9th Python in Science Conference. Austin, TX, 51-56.

McMurdie PJ, Holmes S. 2013. phyloseq: an R package for reproducible interactive analysis and graphics of microbiome census data. PloS one 8:e61217.

Meadow JF, Altrichter AE, Green JL. 2014. Mobile phones carry the personal microbiome of their owners. PeerJ 2:e447.

National Academies of Sciences, Engineering, and Medicine, National Academy of Engineering, Division on Engineering and Physical Sciences, Health and Medicine Division, Division on Earth and Life Studies, Board on Infrastructure and the Constructed Environment, Board on Environmental Studies and Toxicology, Board on Life Sciences, Committee on 
460

461

462

463

464

465

466

467

468

469

470

471

472

473

474

475

476

477

478

479

480

481

482

Microbiomes of the Built Environment: From Research to Application. 2017. Microbiomes

of the Built Environment: A Research Agenda for Indoor Microbiology, Human Health, and Buildings. National Academies Press.

Oksanen J, Blanchet FG, Kindt R, Legendre P, Minchin PR, O’hara RB, Simpson GL, Solymos P, Stevens MHH, Wagner H. 2011. vegan: Community ecology package. $R$ package version:117-118.

Pedregosa F, Varoquaux G, Gramfort A, Michel V, Thirion B, Grisel O, Blondel M, Prettenhofer P, Weiss R, Dubourg V, Vanderplas J, Passos A, Cournapeau D, Brucher M, Perrot M, Duchesnay É. 2011. Scikit-learn: Machine Learning in Python. Journal of machine learning research: JMLR 12:2825-2830.

Price MN, Dehal PS, Arkin AP. 2009. FastTree: computing large minimum evolution trees with profiles instead of a distance matrix. Molecular biology and evolution 26:1641-1650.

Price MN, Dehal PS, Arkin AP. 2010. FastTree 2--approximately maximum-likelihood trees for large alignments. PloS one 5:e9490.

Rinke C, Schwientek P, Sczyrba A, Ivanova NN, Anderson IJ, Cheng J-F, Darling A, Malfatti S, Swan BK, Gies EA, Dodsworth JA, Hedlund BP, Tsiamis G, Sievert SM, Liu W-T, Eisen JA, Hallam SJ, Kyrpides NC, Stepanauskas R, Rubin EM, Hugenholtz P, Woyke T. 2013. Insights into the phylogeny and coding potential of microbial dark matter. Nature 499:431437.

Sánchez-Osuna M, Barbé J, Erill I. 2017. Comparative genomics of the DNA damage-inducible network in the Patescibacteria. Environmental microbiology 19:3465-3474.

Sievers F, Wilm A, Dineen D, Gibson TJ, Karplus K, Li W, Lopez R, McWilliam H, Remmert M, Söding J, Thompson JD, Higgins DG. 2011. Fast, scalable generation of high-quality 
483 protein multiple sequence alignments using Clustal Omega. Molecular systems biology

$484 \quad 7: 539$.

485 Solden L, Lloyd K, Wrighton K. 2016. The bright side of microbial dark matter: lessons learned 486 from the uncultivated majority. Current opinion in microbiology 31:217-226.

487 Whitman WB, Rainey F, Kämpfer P, Trujillo M, Chun J, DeVos P, Hedlund B, Dedysh S (eds.). 488 2015a. Leptotrichia. In: Bergey’s Manual of Systematics of Archaea and Bacteria.

489 Chichester, UK: John Wiley \& Sons, Ltd, 1-10.

490 Whitman WB, Rainey F, Kämpfer P, Trujillo M, Chun J, DeVos P, Hedlund B, Dedysh S (eds.).

491 2015b. Fusobacterium. In: Bergey's Manual of Systematics of Archaea and Bacteria.

492 Chichester, UK: John Wiley \& Sons, Ltd, 1-18.

493 Wickham H. 2010. ggplot2: Elegant Graphics for Data Analysis. Springer New York.

494

495

496

497

498

499

500

501

502 
503 Figure 1: Alpha diversity of cell phone and shoe samples, calculated by either observed counts or by the Shannon diversity index.

505

506

507

508

509

510

511

512

513

514

515

516

517

518

519

520

521

522

523

524

525

526

527

528

529

530

531

532

533

534

Figure 2: Principal coordinate ( $\mathrm{PCoA}$ ) analysis plot of the rarefied (10K sequences) Bray-Curtis distances of 16S rRNA gene sequence based ASVs for cell phone and shoe samples, colored by sample origin. The line is the bisection of the centroids of the two sample types (phones and shoes).

Figure 3: Split Phyla representation of PCoA ordination of Bray-Curtis distances of rarefied ASV counts. Only ANCOM detected, significant ASVs are represented. ASVs biased toward shoes are on the left, those biased towards phones are on the right.

Figure 4: Plot of geographic distance in miles versus Bray Curtis distance of all pairs of locations, separated by cell phones and shoes. A Mantel test performed on both the data from cell phones and shoes, comparing the geographic distance to the Bray Curtis distance, showed no correlation (simulated p-values of .027 and .005 , respectively).

\section{Supplemental Table 1: Summary of all analyses steps}

Supplemental Table 2: Sample Collection Information. "Age" is a rough approximation based on attendees of the event ( $\mathrm{A}=\mathrm{Adult}, \mathrm{K}=\mathrm{Kid}, \mathrm{M}=\mathrm{Mixed}$ ). " $\mathrm{n}=$ " refers to the number of samples that were actually sequenced. "Event title or location" is how the samples are referenced in the data files.

Supplemental Figure 1: Importance of metadata variables (attribute importance analysis)

Supplemental Table 3: MDM taxa found in cell phones and shoes in this study, divided by Phylum, Class, Order, and Family. Shown in "\%Cell" and "\%Shoe" are the percentages of the samples containing particular ASVs found on cell phones or shoes. "Samples" is the total number of samples containing that ASV (of 2243 samples total). "Total" is the total number of samples in the dataset. "CellReads" and "ShoeReads" count the total number of reads containing ASVs at that taxonomic level, "CellTotal" and "ShoeTotal" count the total number of reads found in samples containing ASVs at that taxonomic level. "CellRelab" and ShoeRlab" measure the relative abundance of those ASVs. 
Figure 1

Alpha diversity of cell phone and shoe samples, calculated by either observed counts or by the Shannon diversity index

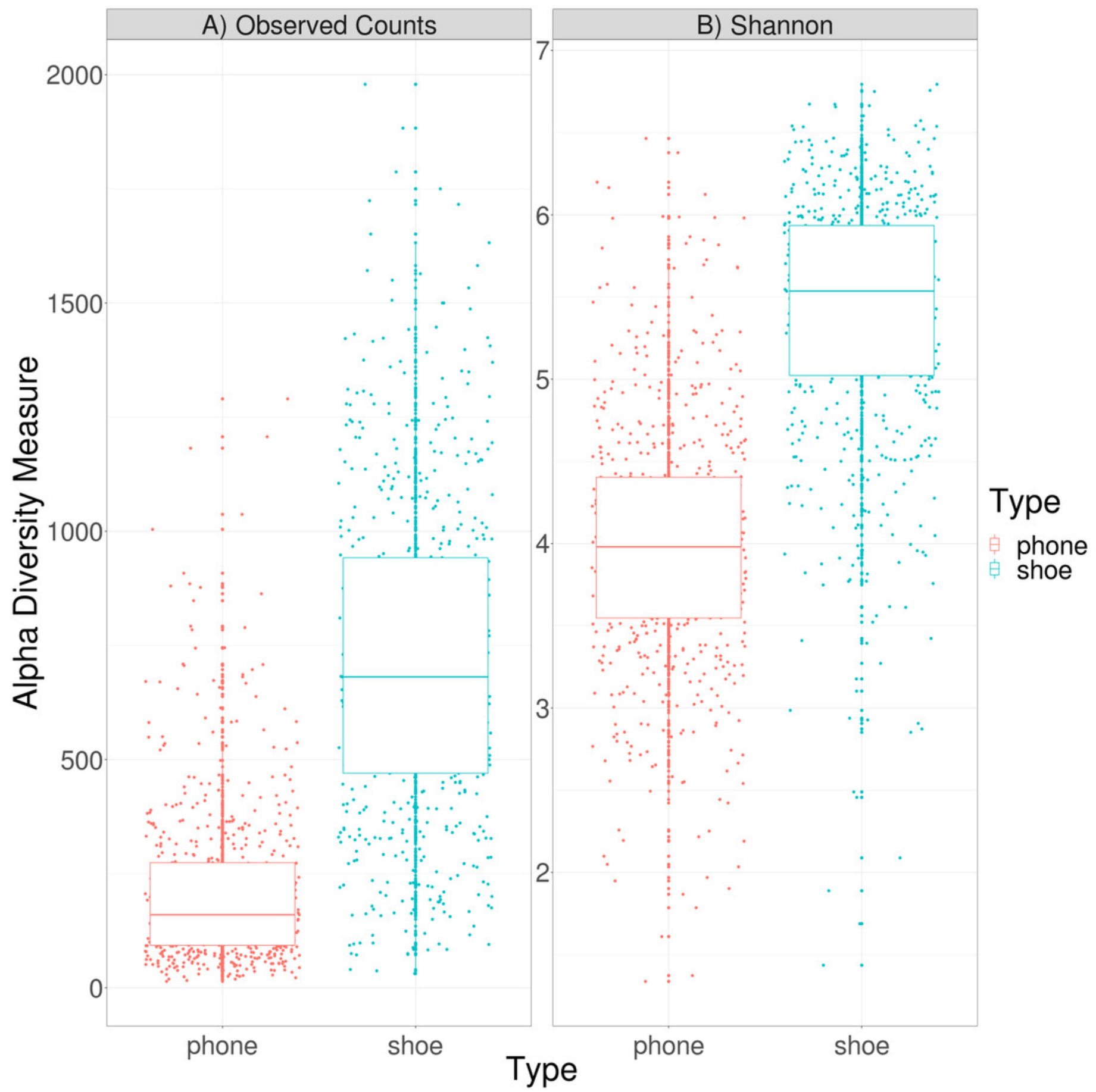


Figure 2

Principal coordinate (PCOA) analysis plot of the rarefied (10K sequences) Bray-Curtis distances of 16S rRNA gene sequence based ASVs for cell phone and shoe samples, colored by sample origin.

The line is the bisection of the centroids of the two sample types (phones and shoes). 


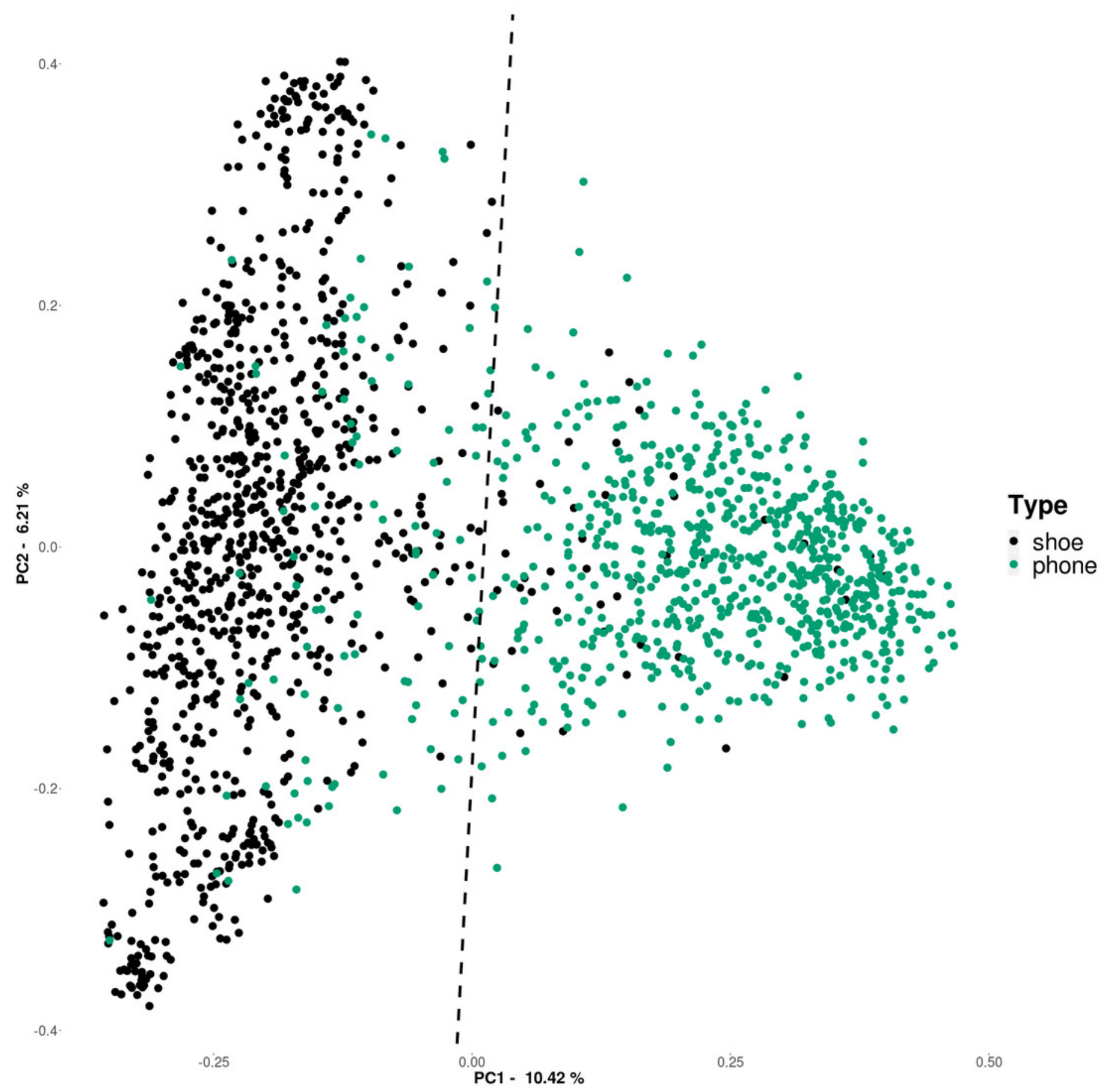


Figure 3

Split Phyla representation of PCoA ordination of Bray-Curtis distances of rarefied ASV counts

Only ANCOM detected, significant ASVs are represented. ASVs biased toward shoes are on the left, those biased towards phones are on the right.
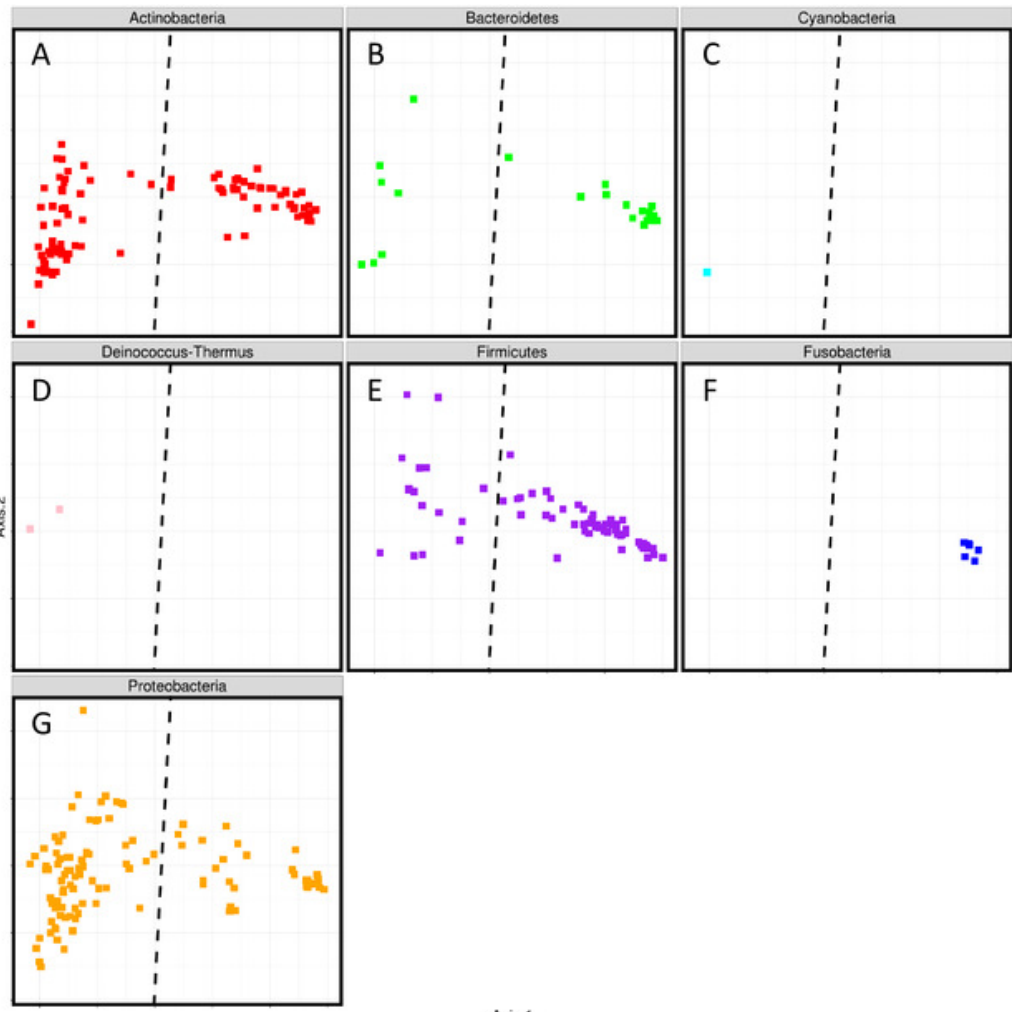

Axis. 1 
Figure 4

Plot of geographic distance in miles versus Bray Curtis distance of all pairs of locations, separated by cell phones and shoes.

A Mantel test performed on both the data from cell phones and shoes, comparing the geographic distance to the Bray Curtis distance, showed no correlation (simulated p-values of .027 and .005 , respectively). 


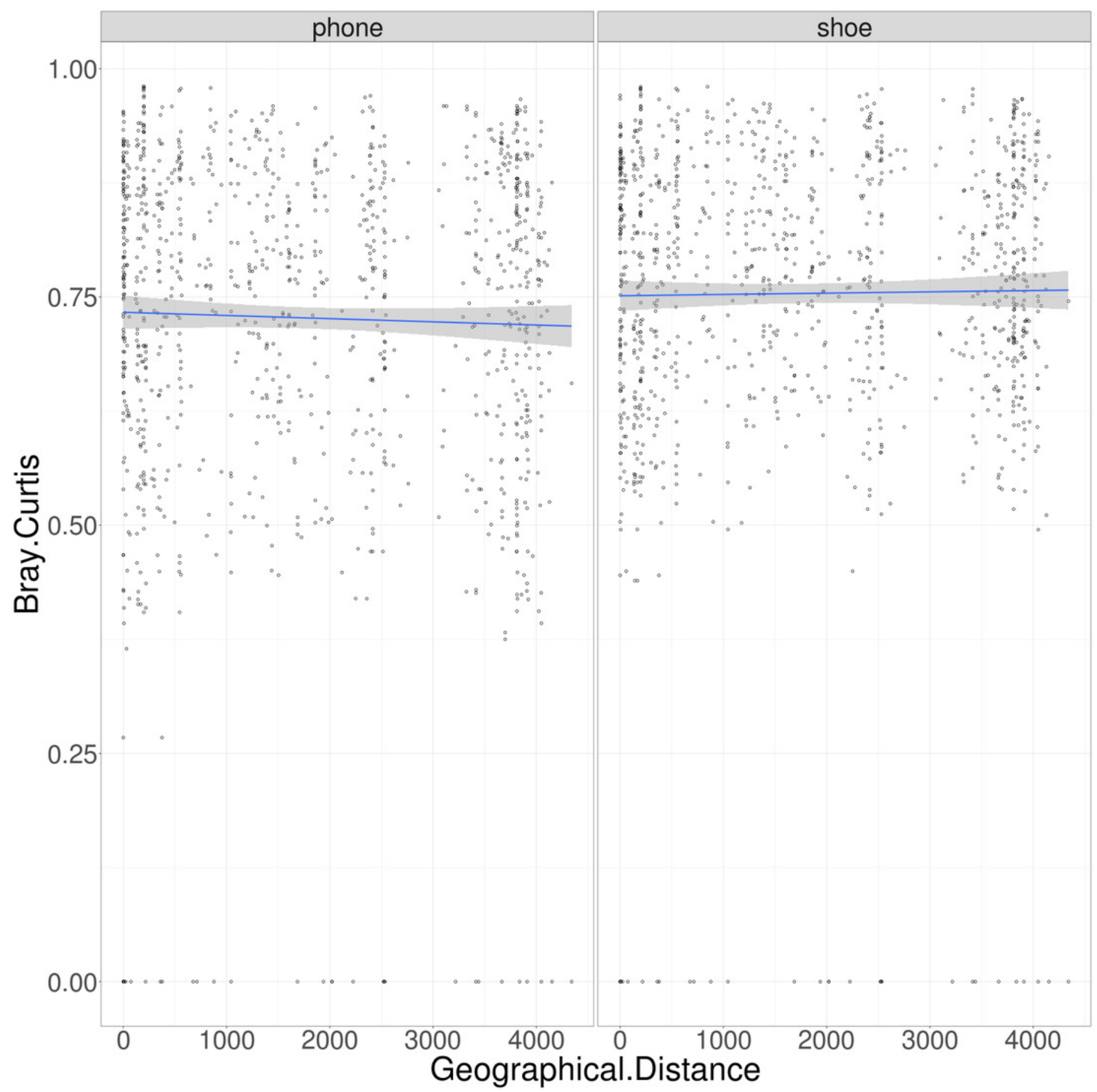

